\title{
Reduced Glucose Tolerance and Skeletal Muscle GLUT4 and IRS1 Content in Cyclists Habituated to a Long-Term Low-Carbohydrate, High-Fat Diet
}

\author{
Christopher C. Webster, Kathryn M. van Boom, Nur Armino, \\ Kate Larmuth, Timothy D. Noakes, and James A. Smith \\ University of Cape Town
}

\author{
Tertius A. Kohn \\ University of Cape Town and \\ University of the Western Cape
}

\begin{abstract}
Very little is known about how long-term ( $>6$ months) adaptation to a low-carbohydrate, high-fat (LCHF) diet affects insulin signaling in healthy, well-trained individuals. This study compared glucose tolerance; skeletal muscle glucose transporter 4 (GLUT4) and insulin receptor substrate 1 (IRS1) content; and muscle enzyme activities representative of the main energy pathways (3-hydroxyacetyl-CoA dehydrogenase, creatine kinase, citrate synthase, lactate dehydrogenase, phosphofructokinase, phosphorylase) in trained cyclists who followed either a long-term LCHF or a mixed-macronutrient (Mixed) diet. On separate days, a 2-hr oral glucose tolerance test was conducted, and muscle samples were obtained from the vastus lateralis of fasted participants. The LCHF group had reduced glucose tolerance compared with the Mixed group, as plasma glucose concentrations were significantly higher throughout the oral glucose tolerance test and serum insulin concentrations peaked later (LCHF, 60 min; Mixed, $30 \mathrm{~min}$ ). Whole-body insulin sensitivity was not statistically significantly different between groups (Matsuda index: LCHF, $8.7 \pm 3.4$ vs. Mixed, $12.9 \pm 4.6 ; p=.08$ ). GLUT4 (LCHF: $1.13 \pm 0.24$; Mixed: $1.44 \pm 0.16 ; p=.026)$ and IRS1 (LCHF: $0.25 \pm 0.13$; Mixed: $0.46 \pm 0.09 ; p=.016)$ protein content was lower in LCHF muscle, but enzyme activities were not different. We conclude that well-trained cyclists habituated to an LCHF diet had reduced glucose tolerance compared with matched controls on a mixed diet. Lower skeletal muscle GLUT4 and IRS1 contents may partially explain this finding. This could possibly reflect an adaptation to reduced habitual glucose availability rather than the development of a pathological insulin resistance.
\end{abstract}

Keywords: glucose clearance, insulin signaling pathway, ketogenic diet

There is growing interest in low-carbohydrate, high-fat (LCHF) diets $(\sim 50 \mathrm{~g} /$ day of carbohydrate) for their potential to improve insulin resistance and type 2 diabetes (T2D) (Bueno et al., 2013; Feinman et al., 2015; Hallberg et al., 2019). Within athletic populations, an increasing number of individuals are experimenting with LCHF diets because of body composition and/or to improve fat oxidation (Gregory et al., 2017; Michalczyk et al., 2018; Volek et al., 2016; Webster et al., 2016). However, LCHF diets are partly controversial because many commonly eaten LCHF foods have been implicated in heart disease, obesity, and T2D (Ludwig et al., 2018). A few days of consuming the LCHF diet can increase postprandial blood glucose concentrations in healthy individuals and reduce the capacity of skeletal muscle to oxidize a carbohydrate load, suggesting a typical state of poor glucose clearance rates synonymous with tissue insulin resistance (Bisschop et al., 2001; Himsworth, 1934, 1940; Numao et al., 2012). Although Himsworth (1934) showed that the LCHF diet causes reduced blood glucose clearance in healthy untrained men, the molecular mechanisms have yet to be established.

Insulin receptor substrate 1 (IRS1) and glucose transporter 4 (GLUT4) are key proteins in the insulin signaling pathway of skeletal muscle. The binding of insulin to the insulin receptor

Webster, van Boom, Armino, Larmuth, Noakes, Smith, and Kohn are with the Division of Exercise Science and Sports Medicine, Department of Human Biology, University of Cape Town, Newlands, South Africa. Kohn is also with the Department of Medical Bioscience, Faculty of Natural Sciences, University of the Western Cape, Bellville, South Africa. Kohn (tkohn@uwc.ac.za) is corresponding author. results in IRS1 phosphorylation, which initiates a signaling cascade and GLUT4 translocation to the membrane and the rapid uptake of glucose (Copps \& White, 2012). During a bout of exercise, however, GLUT4 translocation and its upregulation are regulated via the $\mathrm{Ca}^{2+}$-calmodulin kinase pathways, independent of insulin (Holloszy, 2011; Richter \& Hargreaves, 2013). Impairments in the insulin signaling pathways are associated with insulin resistance and impaired glucose tolerance. The insulin signaling pathway has yet to be investigated after chronic carbohydrate restriction in healthy, well-trained individuals.

The aim of this study was, therefore, to compare glucose tolerance, muscle GLUT4 and IRS1 content, and key enzyme activities representative of major energy pathways, in well-trained cyclists who had followed either a long-term LCHF or a mixedmacronutrient diet (Mixed). We hypothesized that the LCHF group would have lower glucose tolerance, lower GLUT4 and IRS1 content, and higher fat oxidation enzyme activities.

\section{Methods}

The human research ethics committee of the UCT Faculty of Health Sciences approved the study (REF: 453/2012) and was performed in accordance with the principles of the Declaration of Helsinki. Participants provided written informed consent before study enrolment. This cross-sectional analysis formed part of a larger study comparing muscle metabolism, substrate oxidation, and endogenous glucose production (EGP) in trained cyclists habituated to an LCHF diet and matched cyclists habituated to a Mixed 
diet (Webster et al., 2016). For convenience, relevant methods and results from the earlier publication are repeated in this article.

The LCHF and Mixed diet groups each comprised seven healthy, well-trained, male cyclists who were matched for age, $\mathrm{VO}_{2} \mathrm{max}$, peak power output, and body composition (Table 1) and who were following their respective diets without any significant changes for at least 6 months. The mean carbohydrate intake and following duration of the LCHF diet averaged $50 \pm 20 \mathrm{~g} /$ day (range: 5-82 g/day) and 13 \pm 6 months (range: 8-24 months), respectively. Dietary carbohydrate content of the Mixed group was $394 \pm 102 \mathrm{~g} /$ day (range: 272-561 g/day), and they had followed their diets for $107 \pm 115$ months (range: 9-360 months). There was no difference in protein intake or total energy intake between groups (Webster et al., 2016).

The larger study consisted of four laboratory visits on separate days: (a) a peak power output test; (b) an exercise familiarization trial; (c) an experimental trial to measure EGP, muscle glycogen usage, and substrate oxidation; and (d) an oral glucose tolerance test (OGTT). Data from muscle samples collected at rest before the experimental trial and from the OGTT are presented in this paper. Participants completed the experimental trial $72 \mathrm{hr}$ after the familiarization ride ( $2 \mathrm{hr}$ of steady-state cycling at $55 \%$ of peak power output) and were instructed to refrain from exercise during this period. For the experimental trial, participants arrived at the laboratory at 6:00 a.m. in the morning of the experimental trial, following an 11.5-hr overnight fast. A muscle biopsy was performed on the right vastus lateralis at 8.20 a.m., which was $10 \mathrm{~min}$ prior to the start of exercise (Webster et al., 2016). The OGTT was performed on a separate visit, $72 \mathrm{hr}$ after the experimental trial. Participants were instructed to refrain from exercise during this period and arrived at the laboratory on the morning of the OGTT after a 12-hr overnight fast. For the OGTT, a baseline blood sample was collected from a cannula inserted into an antecubital vein, followed by the ingestion of 75-g glucose dissolved in 250-ml water. Blood samples were collected 30-, 60-, 90-, and 120-min postingestion. Blood samples were collected in vacuum tubes (Greiner Bio-One, Kremsmünster, Austria; plasma: oxalate and fluoride; serum: clot activator and gel for serum separation); centrifuged immediately after the trial for $10 \mathrm{~min}$ at $3000 \mathrm{rpm}$; and analyzed for plasma glucose and serum insulin concentrations (glucose oxidase method, YSI 2300 STAT PLUS, New York, NY; $\mathrm{OH}$ and Automated chemiluminescence, Centaur CP system, Siemens Healthcare Diagnostics NY, USA, respectively). The homeostatic model assessment of insulin resistance (HOMA-IR) and Matsuda index were calculated from the OGTT as follows:

$$
\text { HOMA-IR }=\frac{(\text { FPG } \times \text { FSI })}{22.5},
$$

where FPG is fasting plasma glucose (in mmol/L) and FSI is fasting serum insulin (in $\mu \mathrm{U} / \mathrm{ml}$ ).

$$
\text { Matsuda index }=\frac{10,000}{\sqrt{(\text { FPG } \times \text { FSI } \times \mathrm{G} \times \mathrm{I})}},
$$

where $\mathrm{G}$ and $\mathrm{I}$ are the mean glucose and insulin concentrations during the OGTT, respectively (Matsuda \& DeFronzo, 1999). Higher values indicate better whole-body insulin sensitivity.

The hepatic insulin sensitivity index (ISI) was calculated from rates of EGP and fasting insulin concentrations measured at rest during the experimental trial (Webster et al., 2016). The formula was:

$$
\text { Hepatic ISI }=\frac{100}{(\mathrm{EGP} \times \mathrm{FSI})}
$$

where EGP in $\mathrm{mg} / \mathrm{kg} / \mathrm{min}$. Higher values indicate better hepatic insulin sensitivity at rest.

For the quantification of muscle protein content, approximately 50-mg muscle was homogenized and sonicated on ice in a homogenizing buffer containing 56-mM Tris, $\mathrm{pH} 7.4,150-\mathrm{mM}$ $\mathrm{NaCl}, 1-\mathrm{mM}$ EDTA, 0.1\% SDS, 10-mM Na${ }_{4} \mathrm{P}_{2} \mathrm{O}_{7}, 20-\mathrm{mM} \mathrm{NaF}, 1 \mathrm{x}$ complete protease inhibitor cocktail (Roche, Basel, Switzerland), and $1 \%$ Triton-X100. After determining the protein concentration (Bradford, 1976), a volume of sample was diluted with Laemmli sample buffer (ratio 1:1) and loaded in duplicate (with two samples serving as loading controls) onto sodium dodecyl sulfate polyacrylamide gel electrophoresis (SDS-PAGE) gels. GLUT4 (\#ab654, dilution 1:1000; Abcam, Cambridge, MA) and IRS1 (\#3407, 1:1000; Cell Signaling Technology, Danvers, MA) antibody binding linearity were determined by resolving various amounts of total protein and plotting the optical densities of the bands against total protein loaded (Figure 1a, 1b, 1e, and 1f)

\begin{tabular}{|c|c|c|c|}
\hline & LCHF & Mixed & $p$ value \\
\hline \multicolumn{4}{|l|}{ Physiological characteristics } \\
\hline Age (years) & $36 \pm 6$ & $32 \pm 5$ & .24 \\
\hline Weight (kg) & $78 \pm 9$ & $74 \pm 8$ & .41 \\
\hline BMI $\left(\mathrm{kg} / \mathrm{m}^{2}\right)$ & $23.6 \pm 1.8$ & $23.4 \pm 2.0$ & .76 \\
\hline $\mathrm{VO}_{2} \max (\mathrm{ml} / \mathrm{min} / \mathrm{kg})$ & $61 \pm 5$ & $63 \pm 8$ & 61 \\
\hline PPO (W/kg) & $4.8 \pm 0.4$ & $5.0 \pm 0.4$ & .34 \\
\hline \multicolumn{4}{|l|}{ Metabolic parameters } \\
\hline Endogenous glucose production $(\mathrm{mg} / \mathrm{kg} / \mathrm{min})$ & $1.6 \pm 0.2$ & $2.0 \pm 0.3$ & .004 \\
\hline Hepatic insulin sensitivity index & $15.6 \pm 3.9$ & $10.4 \pm 5.0$ & .053 \\
\hline Fat oxidation rate $(\mathrm{g} / \mathrm{min})$ & $1.21 \pm 0.15$ & $0.56 \pm 0.17$ & $<.01$ \\
\hline Carbohydrate oxidation rate (g/min) & $1.22 \pm 0.42$ & $2.89 \pm 0.41$ & $<.01$ \\
\hline
\end{tabular}
(Murphy \& Lamb, 2013). Muscle GLUT4 content was determined

Table 1 Physiological and Metabolic Parameters of Participants 
(a)

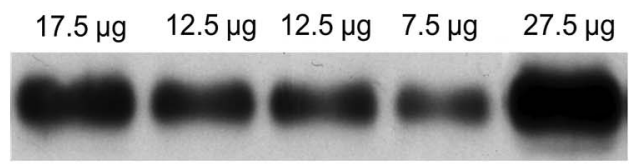

(b)

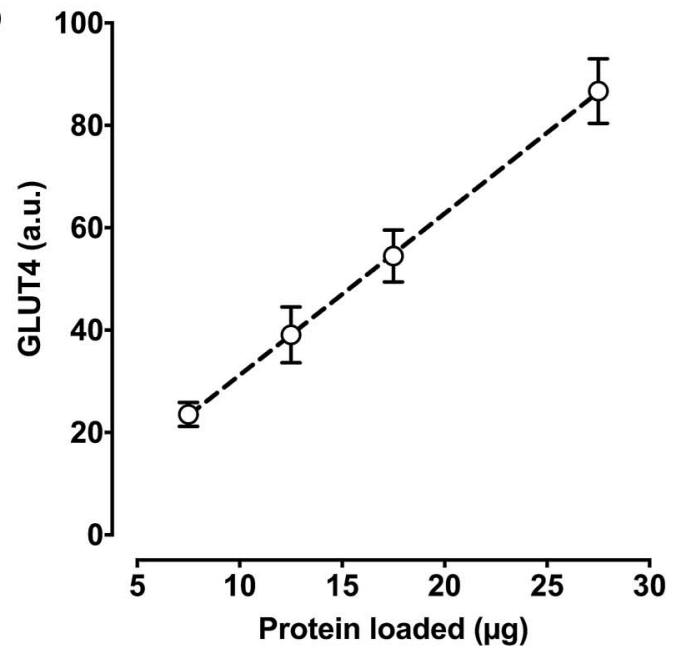

(c)

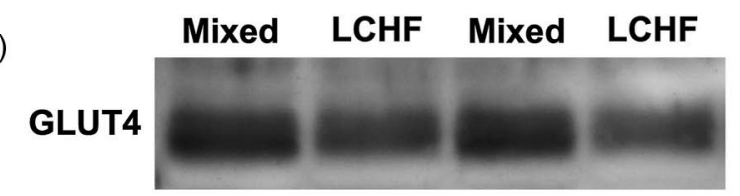

$\alpha$-Tubulin

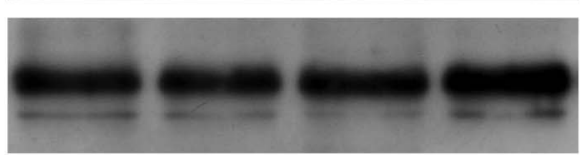

(d)

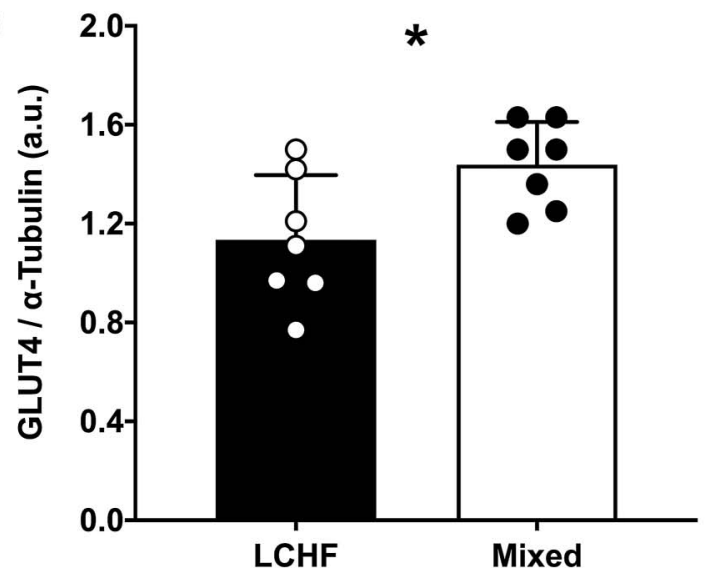

(e)

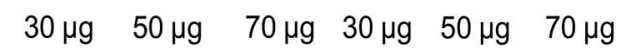

(f)

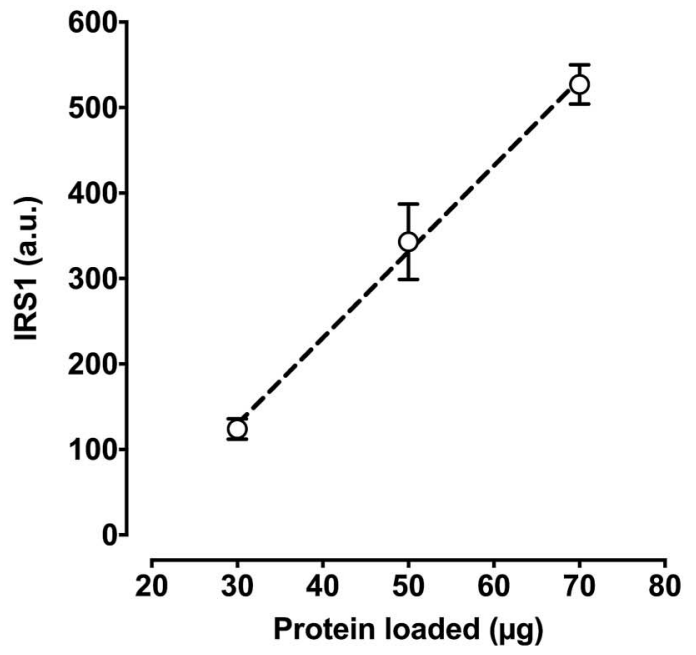

(g)

\section{Mixed LCHF Mixed LCHF}

IRS1

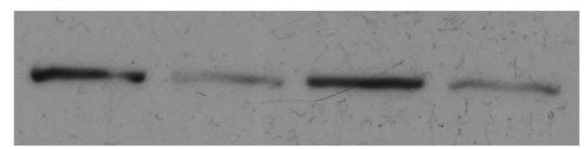

$\alpha$-Tubulin

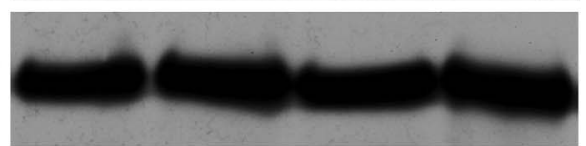

(h)

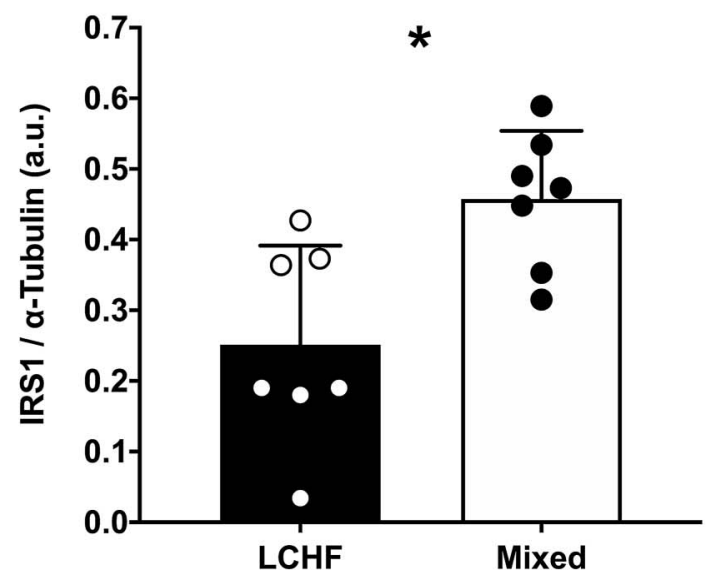

Figure 1 - (a) GLUT4 immunoblot showing respective intensities of various protein concentrations loaded to validate antibody linearity. (b) GLUT4 standard curve plotted from band intensities in (a) to protein concentrations. (c) Representative GLUT4 and $\alpha$-tubulin blots of two LCHF and two Mixed diet athletes. (d) Skeletal muscle GLUT4 content normalized to $\alpha$-tubulin from the two groups. (e) IRS1 immunoblot showing respective intensities of various protein concentrations loaded to validate antibody linearity. (f) IRS1 standard curve plotted from band intensities in (d) to protein concentrations. (g) Representative IRS1 and $\alpha$-tubulin blots of two LCHF and two Mixed diet athletes. (h) Skeletal muscle IRS1 content normalized to $\alpha$-tubulin from the two groups. LCHF indicates low carbohydrate high fat; a.u. = arbitrary units; GLUT4= glucose transporter 4; IRS1 = insulin receptor substrate 1 . *Significant difference between groups $(p<.05)$. $p$ values were determined using the Mann-Whitney $U$ test.

by loading $25-\mu \mathrm{g}$ total protein onto a $10 \%$ denaturing acrylamide gel without heating the sample, whereas the IRS1 sample was heated $\left(5 \mathrm{~min}\right.$ at $\left.95{ }^{\circ} \mathrm{C}\right)$, and $50-\mu \mathrm{g}$ total protein resolved on a $7.5 \%$ gel. The separated proteins were transferred to polyvinylidene difluoride (PVDF) membranes, and blocked in 3\% bovine serum albumin (BSA) phosphate buffered saline (PBS) containing $0.1 \%$ Tween-20 (PBS-T), pH 7.4. Each membrane was incubated in the respective primary antibody diluted in PBS-T overnight at $4{ }^{\circ} \mathrm{C}$. 
After washing, the membranes were incubated with goat anti-rabbit horseradish peroxidase-conjugated secondary antibody for an hour at room temperature. The bands were visualized using chemiluminescence and quantified using Un-Scan-It (Silk Scientific Corporation, Orem, UT) (Figure 1a, 1c, 1e, and 1g). Bands were normalized to $\alpha$-tubulin (\#2144, 1:2000; Cell Signaling Technology) using the same membranes stripped of all antibodies before repeating the steps previously described.

Enzyme activities that served as markers for the respective metabolic pathways were creatine kinase (EC2.7.3.2) for rapid adenosine triphosphate (ATP) replenishment capacity, phosphorylase (EC2.4.1.1) for glycogen breakdown capacity, phosphofructokinase-1 (EC2.7.1.11) for flux capacity through glycolysis, lactate dehydrogenase (EC1.1.1.27) for lactate production from pyruvate, citrate synthase (EC2.3.3.1) for Krebs cycle oxidative capacity, and 3-hydroxyacetyl-CoA dehydrogenase (3HAD; EC1.1.1.35) for fat oxidation capacity. Enzyme activities were determined fluorometrically at excitation and emission wavelengths of 340 and $460 \mathrm{~nm}$, respectively (Kohn et al., 2007; 2011). Briefly, freeze-dried tissue was weighed and homogenized in ice-cold potassium phosphate buffer ( $\mathrm{pH} 7.40)$, sonicated, the protein content determined (as previously described), and stored at $-87{ }^{\circ} \mathrm{C}$ until analyses. A standard nicotinamide adenine dinucleotide (NADH) or nicotinamide adenine dinucleotide phosphate (NADPH) curve was constructed to determine the concentration of produced (reduced) or used (oxidized) pyridine nucleotides in each reaction. For each reaction, the maximum slope was determined and expressed relative to the amount of protein $(\mu \mathrm{mol} / \mathrm{min} / \mathrm{g}$ protein). All reagents were from Sigma-Aldrich (St. Louis, MO), unless otherwise reported.

The relative muscle fiber type was determined by separating the three myosin heavy chain isoforms I, IIA, and IIX using SDS-PAGE, as previously described (Kohn \& Myburgh, 2006). The bands were visualized using silver stain and quantified using Un-Scan-It (Silk Scientific Corporation).

All data are expressed as mean $\pm S D$. Statistical procedures were performed using GraphPad Prism (version 8; GraphPad Software Inc., San Diego, CA). Prior to significance testing, the assumptions of normality and equivalence of variance were tested using the Shapiro-Wilk and Levene tests, respectively. Statistical differences in plasma glucose and serum insulin concentrations during the OGTT were calculated using a mixed-model analysis of variance. If a significant interaction effect was found, further analysis was conducted using Dunnett post hoc test. Insulin sensitivity indices, protein content, and enzyme activities were not normally distributed and nonparametric tests for significance were used. Differences between groups (mean ranks) were determined using the MannWhitney $U$ test. Cohen $d$ is reported as a measure of effect size for the difference in IRS1 and GLUT4 content, and their associations with blood glucose concentrations during the OGTT were determined using Spearman rho. Significance was set at $p<.05$.

\section{Results}

Participant characteristics and relevant results from Webster et al. (2016) are shown in Table 1. Hepatic ISI was not different between groups. Reported substrate oxidation rates were measured during the final 30 min of a 2-hr steady-state ride at $50 \%$ of $\mathrm{VO}_{2}$ max. The LCHF group had significantly higher fat oxidation rates and lower carbohydrate oxidation rates than the Mixed group (Table 1). During the OGTT, there were no differences between groups in fasting plasma glucose or fasting serum insulin concentrations, HOMA-IR (LCHF, $1.0 \pm 0.5$; Mixed, $0.9 \pm 0.4 ; p=.58$ ), or the Matsuda index
(LCHF, $8.7 \pm 3.4$; Mixed, 12.9 $\pm 4.6 ; p=.08$ ). Every individual was within the healthy range for the previous indices (Matsuda \& DeFronzo, 1999; Radikova et al., 2006). Plasma glucose concentrations were higher in the LCHF group during the course of the OGTT (Figure 2a). Peak serum insulin concentrations were similar between the LCHF and Mixed groups $(44.8 \pm 20.2$ vs. $38.6 \pm 26.0 \mu \mathrm{U} / \mathrm{ml}$, respectively), but the peak was significantly later (60 min) in the LCHF group. Serum insulin concentrations had returned to values similar to baseline after $60 \mathrm{~min}$ in the Mixed group, but only after $120 \mathrm{~min}$ in the LCHF group.

The western blot standard curves demonstrate that the binding of the primary antibody to its target fell within the linear concentration range for GLUT4 (Figure 1a and 1b) and IRS1 (Figure 1e and 1f). The GLUT4 (Cohen $d=1.4$ ) and IRS1 (Cohen $d=1.7$ ) content were lower in the LCHF group compared with the Mixed group (Figure 1d and 1h). GLUT4 content correlated negatively with the OGTT plasma glucose concentration area under the curve (Spearman rho $=-.60, p=.027)$, but there were no associations of OGTT parameters with IRS1 content.

There were no differences between groups in the activities of any of the enzymes that were measured (Table 2). Relative muscle fiber type, determined from the myosin heavy chain isoform content, showed a predominance in type I fibers in both groups $(\sim 50 \%)$, followed by myosin heavy chain IIA fibers, with no difference between the groups (Table 2).

\section{Discussion}

This is the first study to investigate glucose clearance and potential mechanisms thereof in well-trained athletes who followed an LCHF diet for longer than 6 months. During the OGTT, plasma glucose concentrations were higher, and serum insulin concentrations peaked later in the LCHF group than in the Mixed group. These results are in line with findings of reduced glucose tolerance after the LCHF diet from previous studies (Bisschop et al., 2001; Himsworth, 1940; Rosenbaum et al., 2019). Short-term LCHF overfeeding (a few meals) can result in reduced glucose tolerance (Parry et al., 2019). These very short-term changes in glucose tolerance likely represent a different mechanism to that of longterm adaptation, such as in the current study. To our knowledge, the molecular mechanisms of decreased glucose clearance rate during adaptation to the LCHF diet have not yet been studied in humans. An important finding from the current study was that skeletal muscle IRS1 and GLUT4 content were lower in the LCHF group. This suggests that chronic exposure to the LCHF diet may result in a downregulation of the insulin signaling pathway, which could partially explain the impaired glucose tolerance that is commonly observed after adaptation to the LCHF diet. Distinguishing between acute versus chronic physiological adaptation to LCHF diets is an important target for future research.

The OGTT evaluates whole-body glycemic control, which is regulated by the interplay between the rate of glucose appearance, glucose clearance, and endogenous glucose production. Glucose clearance rate at rest is increased when insulin binds to its receptor, activating a cascade of events that leads to the translocation of GLUT4 to the plasma membrane, and rapid uptake of glucose ensues. Himsworth (1934) and others have shown that there may be flaws in the OGTT, as apparently healthy individuals on LCHF diets had slower blood glucose clearances (Bisschop et al., 2001; Moon et al., 2010; Numao et al., 2012; Rosenbaum et al., 2019; Volek et al., 2016). Indeed, the LCHF cyclists in the current study are fit and apparently healthy but show some level of insulin 

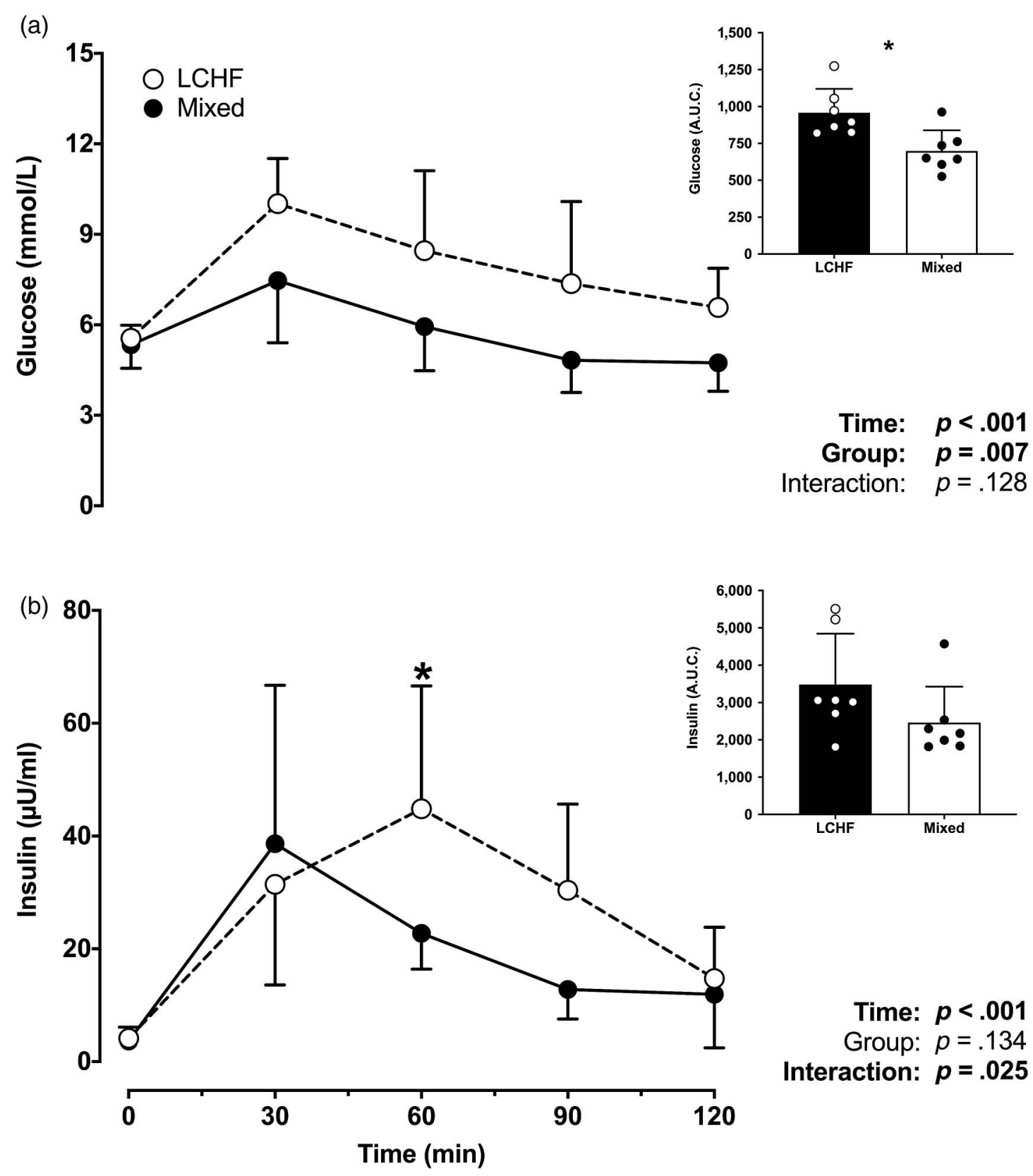

Figure 2 - Concentrations and area under the curve of (a) plasma glucose, and (b) serum insulin during the OGTT. A 75-g glucose bolus was ingested after baseline measures at 0 min. Data are presented as mean $\pm S D ; \mathrm{n}=7$ per group. $p$ values were determined by mixed-model analysis of variance and Dunnett's post hoc test. A.U.C. indicates area under the curve; $\mathrm{LCHF}=$ low carbohydrate high fat; OGTT = oral glucose tolerance test; GLUT4 = glucose transporter 4; IRS1 = insulin receptor substrate $1 .{ }^{*}$ Significant difference between groups $(p<.05)$. Bold values indicate a significant difference.

resistance relative to the Mixed group. The shape of the insulin curve in the LCHF athletes is similar to that of Kraft pattern IIA and Hayashi pattern 3 (peak insulin at $60 \mathrm{~min}$ ), which is associated with an increased risk of developing T2D (DiNicolantonio et al., 2017). However, the magnitude of the insulin peak in the LCHF athletes (at $\sim 45 \mu \mathrm{U} / \mathrm{ml}$ ) best resembles Kraft pattern 1, which is associated with normal health (DiNicolantonio et al., 2017). Although within the healthy ranges, the relative reduced glucose tolerance and the inferior insulin profile of the LCHF group would typically be considered clinically relevant.

The finding that the LCHF cyclists had reduced glucose tolerance does not necessarily imply a pathological insulin resistance. On the contrary, they may merely be unaccustomed to a bolus of ingested glucose. We observed some markers in the LCHF athletes that would be uncharacteristic of individuals with pathological insulin resistance. Impaired fat oxidation was once considered a common feature of pathological insulin resistance (Holloszy, 2013; Hulver et al., 2003; Kelley et al., 1999; Kim et al., 2000). However, the LCHF group in this study had very good fat oxidation capacity. They also had high 3HAD and citrate synthase activities, conforming to that of well-trained endurance athletes (Kohn et al., 2007), and HOMA-IR, the Matsuda index, and hepatic ISI provided no indication of pathologies. This study highlights the importance of further research into the long-term 
Table 2 Enzyme Activities and MHC Isoform Content of the Vastus Lateralis

\begin{tabular}{lccc}
\hline & LCHF & Mixed & $\boldsymbol{p}$ value \\
\hline Activity $(\mu \mathrm{mol} / \mathrm{min} / \mathrm{g}$ prot$)$ & & & \\
CS & $66 \pm 16$ & $56 \pm 17$ & .38 \\
3HAD & $97 \pm 23$ & $88 \pm 18$ & .60 \\
PFK & $163 \pm 52$ & $202 \pm 69$ & .26 \\
LDH & $592 \pm 174$ & $480 \pm 162$ & .32 \\
CK & $11391 \pm 1525$ & $10118 \pm 1162$ & .10 \\
PHOS & $328 \pm 75$ & $345 \pm 98$ & .99 \\
MHC isoform content $(\%)$ & & & \\
MHC I & $50 \pm 8$ & $53 \pm 8$ & .62 \\
MHC IIA & $45 \pm 7$ & $38 \pm 4$ & .07 \\
MHC IIX & $5 \pm 5$ & $9 \pm 9$ & .33 \\
\hline
\end{tabular}

Note. $\mathrm{CS}=$ citrate synthase; $3 \mathrm{HAD}=3$-hydroxyacetyl-CoA dehydrogenase; $\mathrm{LDH}=$ lactate dehydrogenase; $\mathrm{PFK}=$ phosphofructokinase; $\mathrm{CK}=$ creatine kinase; PHOS = myophosphorylase; prot $=$ protein $; \mathrm{MHC}=$ myosin heavy chain .

progression of hyperinsulinemia in individuals who follow the LCHF diet. It is also unclear why the activities of some enzymes appear sensitive to diet, such as pyruvate dehydrogenase and carnitine acyltransferase (Goedecke et al., 1999; Stellingwerff et al., 2006), whereas others such as citrate synthase, 3HAD, and hexokinase appear unaffected (Goedecke et al., 1999). Rates of fat oxidation during exercise were vastly different between athletes in the LCHF and Mixed groups and this seems incongruent with our finding of similar 3HAD activity.

A possible mechanism for the lower glucose tolerance is that chronic exposure to the LCHF diet reduces insulin secretion and circulating insulin concentrations, resulting in reduced insulin receptor activation and decreased expression of GLUT4 and IRS1 proteins (Chibalin et al., 2000). Most recently, a pioneering study by Hancock et al. (2019) unequivocally showed that the insulin receptor, once internalized, translocates from the cell surface to the nucleus, where it can associate with transcriptional machinery at various gene promoters linked to insulin functions. This finding was shown using mouse and human liver tissue and cells. It would be of great value if the translocation of the insulin complex to skeletal muscle nuclei could be investigated in response to various diets and exercise. However, hypothetically, the finding of lower IRS1 protein content could mean that the expression of the insulin receptor complex (e.g., IRS1) is downregulated due to a lack in insulin-mediated promotor binding resulting from the LCHF diet. The long-term outcome of the LCHF diet would be fewer functional insulin receptors and reduced GLUT4 content, which would reduce the efficiency to take up a glucose bolus through the insulin-dependent glucose transport system. It must be acknowledged that, apart from the previous explanation, insulin secretion and insulin extraction by the liver may also be affected as a direct consequence of the LCHF diet and the role of these tissues requires further investigation using tracer studies.

This study is limited by small sample size, and there are potential confounders related to habitual diet and physical activity due to the cross-sectional design. The amount of muscle tissue was limited, so we could not measure other markers of the insulin pathway such as skeletal muscle insulin receptors or GLUT4 translocation. A future cross-over long-term diet trial (LCHF vs. Mixed diet) using healthy untrained and trained individuals should measure glucose oxidation, insulin secretion changes, and phosphorylation states of proteins involved in the insulin signaling pathway during an OGTT.

In conclusion, this study found that cyclists adapted to a longterm LCHF diet had reduced glucose tolerance and a delayed insulin peak during an OGTT. In addition, IRS1 and GLUT4 content were lower in the LCHF group, providing a potential mechanism that partly explains the lower rate of blood glucose clearance. This likely reflects an adaptation to reduced habitual glucose availability rather than indicating pathological insulin resistance.

\section{Acknowledgments}

A heartfelt thank you is extended to Hendriena Victor for performing the glucose and insulin sample analyses. Conceptualization and design: J.A. Smith, T.D. Noakes, and T.A. Kohn; funding acquisition: J.A. Smith and T.D. Noakes; sample collection: T.A. Kohn, C.C. Webster, and J.A. Smith; sample analysis: T.A. Kohn, K.M. van Boom, N. Armino, and K. Larmuth; data analysis: C.C. Webster and T.A. Kohn; writing: C.C. Webster, K.M. van Boom, T.A. Kohn, and J.A. Smith; reviewing and editing: all authors. All authors approved the final version of this manuscript and agreed to be accountable for all aspects thereof. This study was funded by a grant allocated to T.D. Noakes and J.A. Smith from the National Research Foundation of South Africa RCA13100251066, grant number 91443. T.A. Kohn is a recipient of the Tim and Marilyn Noakes Sports Science Postdoctoral Fellowship. T.D. Noakes is the author of the books Lore of Running and Waterlogged and co-author of The Real Meal Revolution, Challenging Beliefs, Raising Superheroes (Superfoods for Superheroes), Lore of Nutrition (Real Food on Trial), and The Banting Pocket Guide. All royalties from the sale of books dealing with nutrition are donated to The Noakes Foundation of which he is the chairman and which funds research of insulin resistance, diabetes, and nutrition (including this study) as directed by its Board of Directors. Money from the sale of other books is donated to the Tim and Marilyn Noakes Sports Science Research Trust. The other authors have no conflicts to declare.

\section{References}

Bisschop, P.H., de Metz, J., Ackermans, M.T., Endert, E., Pijl, H., Kuipers, F., ... Romijn, J.A. (2001). Dietary fat content alters insulinmediated glucose metabolism in healthy men. The American Journal of Clinical Nutrition, 73(3), 554-559. PubMed ID: 11237931 doi:10. 1093/ajen/73.3.554

Bradford, M.M. (1976). A rapid and sensitive method for the quantitation of microgram quantities of protein utilizing the principle of proteindye binding. Analytical Biochemistry, 72(1/2), 248-254. PubMed ID: 942051 doi:10.1016/0003-2697(76)90527-3

Bueno, N.B., de Melo, I.S., de Oliveira, S.L., \& da Rocha Ataide, T. (2013). Very-low-carbohydrate ketogenic diet v. low-fat diet for long-term weight loss: A meta-analysis of randomised controlled trials. The British Journal of Nutrition, 110(7), 1178-1187. PubMed ID: 23651522 doi:10.1017/S0007114513000548

Chibalin, A.V., Yu, M., Ryder, J.W., Song, X.M., Galuska, D., Krook, A., ... Zierath, J.R. (2000). Exercise-induced changes in expression and activity of proteins involved in insulin signal transduction in skeletal muscle: Differential effects on insulin-receptor substrates 1 and 2. Proceedings of the National Academy of Sciences of the United States of America, 97(1), 38-43. PubMed ID: 10618367 doi:10.1073/pnas.97.1.38

Copps, K.D., \& White, M.F. (2012). Regulation of insulin sensitivity by serine/threonine phosphorylation of insulin receptor substrate proteins IRS1 and IRS2. Diabetologia, 55(10), 2565-2582. PubMed ID: 22869320 doi:10.1007/s00125-012-2644-8 
DiNicolantonio, J.J., Bhutani, J., OKeefe, J.H., \& Crofts, C. (2017). Postprandial insulin assay as the earliest biomarker for diagnosing pre-diabetes, type 2 diabetes and increased cardiovascular risk. Open Heart, 4(2), e000656. PubMed ID: 29225902 doi:10.1136/openhrt2017-000656

Feinman, R.D., Pogozelski, W.K., Astrup, A., Bernstein, R.K., Fine, E.J., Westman, E.C., ... McFarlane, S.I. (2015). Dietary carbohydrate restriction as the first approach in diabetes management: Critical review and evidence base. Nutrition 31(1), 1-13. PubMed ID: 25287761 doi:10.1016/j.nut.2014.06.011

Goedecke, J.H., Christie, C., Wilson, G., Dennis, S.C., Noakes, T.D., Hopkins, W.G., \& Lambert, E.V. (1999). Metabolic adaptations to a high-fat diet in endurance cyclists. Metabolism, 48(12), 1509-1517. PubMed ID: 10599981 doi:10.1016/S0026-0495(99)90238-X

Gregory, R.M., Hamdan, H., Torisky, D.M., \& Akers, J.D. (2017). A lowcarbohydrate ketogenic diet combined with 6-weeks of crossfit training improves body composition and performance. International Journal of Sports and Exercise Medicine, 3(2), 054. doi:10.23937/ 2469-5718/1510054

Hallberg, S.J., Gershuni, V.M., Hazbun, T.L., \& Athinarayanan, S.J. (2019). Reversing type 2 diabetes: A narrative review of the evidence. Nutrients, 11(4), E766. PubMed ID: 30939855 doi:10.3390/ nu11040766

Hancock, M.L., Meyer, R.C., Mistry, M., Khetani, R.S., Wagschal, A., Shin, T., .. Flanagan, J.G. (2019). Insulin receptor associates with promoters genome-wide and regulates gene expression. Cell, 177(3), 722-736.e22. PubMed ID: 30955890 doi:10.1016/j.cell. 2019.02.030

Himsworth, H.P. (1934). High carbohydrate diets and insulin efficiency. British Medical Journal, 2(3836), 57-60. PubMed ID: 20778390 doi:10.1136/bmj.2.3836.57

Himsworth, H.P. (1940). Insulin deficiency and insulin inefficiency. British Medical Journal, 1(4139), 719-722. PubMed ID: 20783080 doi:10.1136/bmj.1.4139.719

Holloszy, J.O. (2011). Regulation of mitochondrial biogenesis and GLUT4 expression by exercise. Comprehensive Physiology, 1(2), 921-940. PubMed ID: 23737207 doi:10.1002/cphy.c100052

Holloszy, J.O. (2013). "Deficiency" of mitochondria in muscle does not cause insulin resistance. Diabetes, 62(4), 1036-1040. PubMed ID: 23520283 doi:10.2337/db12-1107

Hulver, M.W., Berggren, J.R., Cortright, R.N., Dudek, R.W., Thompson, R.P., Pories, W.J., . . Houmard, J.A. (2003). Skeletal muscle lipid metabolism with obesity. American Journal of Physiology, Endocrinology and Metabolism, 284(4), E741-E747. PubMed ID: 12626325 doi:10.1152/ajpendo.00514.2002

Kelley, D.E., Goodpaster, B., Wing, R.R., \& Simoneau, J.A. (1999). Skeletal muscle fatty acid metabolism in association with insulin resistance, obesity, and weight loss. The American Journal of Physiology, 277(6), E1130-E1141. PubMed ID: 10600804 doi:10.1152/ ajpcell.1999.277.6.C1130

Kim, J.Y., Hickner, R.C., Cortright, R.L., Dohm, G.L., \& Houmard, J.A. (2000). Lipid oxidation is reduced in obese human skeletal muscle. American Journal of Physiology, Endocrinology and Metabolism, 279(5), E1039-E1044. PubMed ID: 11052958 doi:10.1152/ajpcell. 2000.279.2.C326

Kohn, T.A., Burroughs, R., Hartman, M.J., \& Noakes, T.D. (2011). Fiber type and metabolic characteristics of lion (Panthera leo), caracal (Caracal caracal) and human skeletal muscle. Comparative Biochemistry and Physiology. Part A Molecular and Integrative Physiology, 159(2), 125133. PubMed ID: 21320626 doi:10.1016/j.cbpa.2011.02.006

Kohn, T.A., Essen-Gustavsson, B., \& Myburgh, K.H. (2007). Do skeletal muscle phenotypic characteristics of Xhosa and Caucasian endurance runners differ when matched for training and racing distances? Journal of Applied Physiology, 103(3), 932-940. PubMed ID: 17585041 doi:10.1152/japplphysiol.01221.2006

Kohn, T.A., \& Myburgh, K.H. (2006). Electrophoretic separation of human skeletal muscle myosin heavy chain isoforms: The importance of reducing agents. The Journal of Physiological Sciences, 56(5), 355-360. PubMed ID: 17014746 doi:10.2170/physiolsci. RP007706

Ludwig, D.S., Willett, W.C., Volek, J.S., \& Neuhouser, M.L. (2018). Dietary fat: From foe to friend? Science, 362(6416), 764-770. PubMed ID: 30442800 doi:10.1126/science.aau2096

Matsuda, M., \& DeFronzo, R.A. (1999). Insulin sensitivity indices obtained from oral glucose tolerance testing: Comparison with the euglycemic insulin clamp. Diabetes Care, 22(9), 1462-1470. PubMed ID: 10480510 doi:10.2337/diacare.22.9.1462

Michalczyk, M., Zajac, A., Mikolajec, K., Zydek, G., \& Langfort, J. (2018). No modification in blood lipoprotein concentration but changes in body composition after 4 weeks of low carbohydrate diet (LCD) followed by 7 days of carbohydrate loading in basketball players. Journal of Human Kinetics, 65(1), 125-137. PubMed ID: 30687425 doi:10.2478/hukin-2018-0102

Moon, J.H., Lee, J.Y., Kang, S.B., Park, J.S., Lee, B.W., Kang, E.S., . . . Cha, B.S. (2010). Dietary monounsaturated fatty acids but not saturated fatty acids preserve the insulin signaling pathway via IRS-1/PI3K in rat skeletal muscle. Lipids, 45(12), 1109-1116. PubMed ID: 20960069 doi:10.1007/s11745-010-3475-3

Murphy, R.M., \& Lamb, G.D. (2013). Important considerations for protein analyses using antibody based techniques: Down-sizing Western blotting up-sizes outcomes. The Journal of Physiology, 591(23), 5823-5831. PubMed ID: 24127618 doi:10.1113/jphysiol.2013. 263251

Numao, S., Kawano, H., Endo, N., Yamada, Y., Konishi, M., Takahashi, M., \& Sakamoto, S. (2012). Short-term low carbohydrate/high-fat diet intake increases postprandial plasma glucose and glucagon-like peptide-1 levels during an oral glucose tolerance test in healthy men. European Journal of Clinical Nutrition, 66(8), 926-931. PubMed ID: 22669333 doi:10.1038/ejen.2012.58

Parry, S.A., Turner, M.C., Woods, R.M., James, L.J., Ferguson, R.A., Cocks, M., ... Wagenmakers, A.J. (2019). High-fat overfeeding impairs peripheral glucose metabolism and muscle microvascular eNOS Ser1177 phosphorylation. The Journal of Clinical Endocrinology and Metabolism, 105(1), dgz018. PubMed ID: 31513265 doi:10.1210/clinem/dgz018

Radikova, Z., Koska, J., Huckova, M., Ksinantova, L., Imrich, R., Vigas, M., ... Klimes, I. (2006). Insulin sensitivity indices: A proposal of cut-off points for simple identification of insulin-resistant subjects. Experimental and Clinical Endocrinology \& Diabetes, 114(5), 249-256. PubMed ID: 16804799 doi:10.1055/s-2006-924233

Richter, E.A., \& Hargreaves, M. (2013). Exercise, GLUT4, and skeletal muscle glucose uptake. Physiological Reviews, 93(3), 993-1017. PubMed ID: 23899560 doi:10.1152/physrev.00038.2012

Rosenbaum, M., Hall, K.D., Guo, J., Ravussin, E., Mayer, L.S., Reitman, M.L., ... Leibel, R.L. (2019). Glucose and lipid homeostasis and inflammation in humans following an isocaloric ketogenic diet. Obesity, 27(6), 971-981. PubMed ID: 31067015 doi:10.1002/oby. 22468

Stellingwerff, T., Spriet, L.L., Watt, M.J., Kimber, N.E., Hargreaves, M., Hawley, J.A., \& Burke, L.M. (2006). Decreased PDH activation and glycogenolysis during exercise following fat adaptation with carbohydrate restoration. American Journal of Physiology, Endocrinology and metabolism, 290(2), E380-E388. PubMed ID: 16188909 doi:10. 1152/ajpendo.00268.2005 
Volek, J.S., Freidenreich, D.J., Saenz, C., Kunces, L.J., Creighton, B.C., Bartley, J.M., ... Phinney, S.D. (2016). Metabolic characteristics of keto-adapted ultra-endurance runners. Metabolism, 65(3), 100-110. PubMed ID: 26892521 doi:10.1016/j.metabol. 2015.10 .028
Webster, C.C., Noakes, T.D., Chacko, S.K., Swart, J., Kohn, T.A., \& Smith, J.A. (2016). Gluconeogenesis during endurance exercise in cyclists habituated to a long-term low carbohydrate high-fat diet. The Journal of Physiology, 594(15), 4389-4405. PubMed ID: 26918583 doi:10.1113/JP271934 